\title{
Correction to: Population pharmacokinetic and exploratory exposure-response analysis of the fixed-dose combination of pertuzumab and trastuzumab for subcutaneous injection in patients with HER2-positive early breast cancer in the FeDeriCa study
}

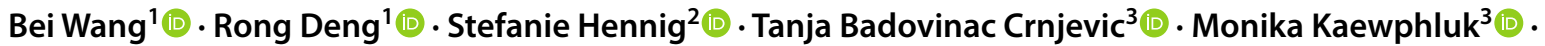

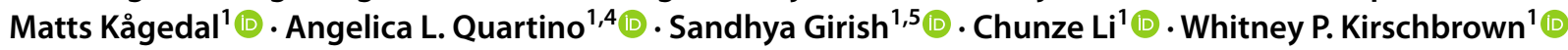

Published online: 24 September 2021

(c) Springer-Verlag GmbH Germany, part of Springer Nature 2021

\section{Correction to: Cancer Chemotherapy and Pharmacology https://doi.org/10.1007/s00280-021-04296-0}

In the original publication, there were errors in some sentences that are corrected as below:

In the keywords section, Page 1, Lines 1-2: the "Pertuzumab", "Trastuzumab", and "Hyaluronidase-zzxf" should all be replaced with "Pertuzumab, trastuzumab, and hyaluronidase-zzxf".

In the Introduction section, Page 2, Paragraph 3, Lines $10-11:$ " $\left(\mathrm{C}_{\text {trough }}\right.$ and area under the curve [AUC] $)$ " should be replaced with " $\left[\mathrm{C}_{\text {trough }}\right.$ and area under the curve (AUC) $]$ ".

In the Introduction section, Page 2, Paragraph 3, Lines 18-19: "2000 U/mL" currently spans over two lines; it should be on the same line.

In the Materials and methods section, Study design, Lines 9-13: "(4 cycles of dose-dense doxorubicin plus cyclophosphamide (ddAC) every 2 weeks followed by paclitaxel once weekly for 12 weeks, or 4 cycles of doxorubicin plus cyclophosphamide (AC) every 3 weeks followed by 4 cycles

The original article can be found online at https://doi.org/10.1007/ s00280-021-04296-0.

Whitney P. Kirschbrown

kirschbrown.whitney@gene.com

1 Genentech, Inc., 1 DNA Way, South San Francisco, CA 94080, USA

2 Certara, Inc., Princeton, NJ, USA

3 F. Hoffmann-La Roche Ltd, Basel, Switzerland

4 Present Address: Clinical Pharmacology and Quantitative Pharmacology, AstraZeneca, Gothenburg, Sweden

5 Present Address: Gilead Sciences, Inc., Foster City, CA, USA of docetaxel every 3 weeks)" should be replaced with "[4 cycles of dose-dense doxorubicin plus cyclophosphamide (ddAC) every 2 weeks followed by paclitaxel once weekly for 12 weeks, or 4 cycles of doxorubicin plus cyclophosphamide (AC) every 3 weeks followed by 4 cycles of docetaxel every 3 weeks]".

In the Materials and methods section, Procedures, Paragraph 1, Lines 11-12: "HER2-targeted" currently spans over two lines; it should be on the same line.

In the Materials and methods section, Procedures, Paragraph 1, Lines 12-13: "P IV" currently spans over two lines; it should be on the same line.

In the Materials and methods section, Pertuzumab population pharmacokinetic analysis, Paragraph 2, Lines 13-19: "[hormone receptor status (estrogen receptor- or progesterone receptor-positive, or estrogen receptor- and progesterone receptor-negative), clinical stage (II-IIIA or IIIB-IIIC), and chemotherapy regimen (4 cycles of ddAC every 2 weeks followed by paclitaxel once weekly for 12 weeks, or 4 cycles of AC every 3 weeks followed by 4 cycles of docetaxel every 3 weeks]" should be replaced with "[hormone receptor status (estrogen receptor- or progesterone receptor-positive, or estrogen receptor- and progesterone receptor-negative), clinical stage (II-IIIA or IIIB-IIIC), and chemotherapy regimen ( 4 cycles of ddAC every 2 weeks followed by paclitaxel once weekly for 12 weeks, or 4 cycles of AC every 3 weeks followed by 4 cycles of docetaxel every 3 weeks)]".

In the Results section, Pertuzumab population pharmacokinetic analysis, Page 4, Paragraph 1, Line 18: "(F)" should be replaced with " $(F)$ ", i.e., ' $F$ ' in italics.

In the Results section, Pertuzumab population pharmacokinetic analysis, Page 4, Paragraph 1, Line 19: 
"CL (L/days) $=0.163$ " should be replaced with "CL (L/ days $)=0.163$ ", i.e., a space inserted after the equals sign.

In the Results section, Pertuzumab population pharmacokinetic analysis, Page 4, Paragraph 1, Line 20: " $\times(1.123$ if" should be replaced with " $\times(1.123$ if", i.e., a space inserted after the ' 1.123 '.

In the Results section, Pertuzumab population pharmacokinetic analysis, Page 4, Paragraph 1, Line 21: " $V_{\mathrm{c}}$ " should be replaced with " $V_{c}$ ", i.e., ' ' ${ }_{c}$ in italics.

In the Results section, Pertuzumab population pharmacokinetic analysis, Page 5, Paragraph 1, Line 1: "Q(L/days)" should be replaced with "Q (L/days)", i.e. a space inserted after the 'Q'.

In the Results section, Pertuzumab population pharmacokinetic analysis, Page 5, Paragraph 1, Line 2: " $V_{\mathrm{p}}$ " should be replaced with " $V_{p}$ ", i.e., ' $p$ in italics.

In the Results section, Pertuzumab population pharmacokinetic analysis, Page 5, Paragraph 1, Lines 1-3:

“ $(\mathrm{Q}, \mathrm{L} /$ days $)=0.616$,

$V_{\mathrm{p}}(\mathrm{L})=2.49 \times(\mathrm{LBW} / 45.09)^{0.716} \times e^{\eta V 3}$,
$F=0.712 \times e^{\eta F}$

" should be moved up to the previous page to keep all equations together.

In the Results section, Exposure-safety of PH FDC SC, Paragraph 1, Lines 7-8: "grade $\geq 3$ " currently spans over two lines; it should be on the same line.

In the Declarations section, Conflict of interest, Lines 8-9: "Stephanie Hennig" currently spans over two lines; it should be on the same line.

The authors sincerely apologize for any inconvenience caused by these errors.

The original article has been corrected.

Publisher's Note Springer Nature remains neutral with regard to jurisdictional claims in published maps and institutional affiliations. 